\title{
(2) OPEN ACCESS \\ Safety and performance of a suprachoroidal sensor for telemetric measurement of intraocular pressure in the EYEMATE-SC trial
}

\author{
Peter Szurman, ${ }^{1}$ Kaweh Mansouri, ${ }^{2}$ H. Burkhard Dick, ${ }^{3}$ Andre Mermoud, ${ }^{2}$ \\ Esther M Hoffmann, ${ }^{4}$ Marc Mackert, ${ }^{5}$ Robert N Weinreb, ${ }^{6}$ Harsha Laxmana Rao (1) , 7,8 \\ Anna-Maria Seuthe $10,{ }^{9}$ On behalf of the EYEMATE-SC study group
}

${ }^{1}$ Eye Clinic Sulzbach, Knappschaft Hospital Saar 66280 Sulzbach, Germany ${ }^{2}$ Glaucoma Research Centre, Montchoisi Hospital, Lausanne, Switzerland

${ }^{3}$ Eye Clinic, University Hospital of the Ruhr University Bochum, Bochum, Germany

${ }^{4}$ Ophthalmology, University Medical Centre of the Johannes Gutenberg University Mainz, Mainz, Germany

${ }^{5}$ Department of Ophthalmology, University Hospital, Ludwig-

Maximilians-Universitat

Munchen Medizinische Fakultat, Munich, Germany

${ }^{6}$ Department of Ophthalmology at the Shiley Eye Institute,

University of California at San Diego, La Jolla, California, USA

Glaucoma, Narayana

Nethralaya, Bangalore, India

${ }^{8}$ Ophthalmology, Maastricht University Medical Centre+, Maastricht, The Netherlands ${ }^{9}$ Eye Clinic Sulzbach, Saar Knappschaft Hospital Sulzbach, Sulzbach, Germany

\section{Correspondence to}

Dr Peter Szurman, Eye Clinic Sulzbach, Knappschaft Hospital Saar, 66280 Sulzbach, Saarland, Germany;

Peter.Szurman@kksaar.de

Received 6 July 2021

Accepted 18 October 2021

Check for updates

(c) Author(s) (or their employer(s)) 2021. Re-use permitted under CC BY-NC. No commercial re-use. See rights and permissions. Published by BMJ.

To cite: Szurman $P$ Mansouri K, Dick HB, et al. Br J Ophthalmol Epub ahead of print: [please include Day Month Year]. doi:10.1136/ bjophthalmol-2021-320023

\begin{abstract}
Aim To investigate the safety and performance of a telemetric suprachoroidal intraocular pressure (IOP) sensor (EYEMATE-SC) and the accuracy of its IOP measurements in open angle glaucoma (OAG) patients undergoing simultaneous non-penetrating glaucoma surgery (NPGS).
\end{abstract}

Methods Prospective, multicentre, open-label, single-arm, interventional clinical trial. Twenty-four eyes of 24 patients with OAG regularly scheduled for NPGS (canaloplasty or deep sclerectomy) were simultaneously implanted with an EYEMATE-SC sensor. Six-month follow-up on the sensor's safety and performance as well as on the level of agreement between the EYEMATE-SC measurements and IOP measurements with Goldmann applanation tonometry (GAT).

Results The eyes underwent canaloplasty $(n=15)$ or deep sclerectomy $(n=9)$ and achieved successful implantation of the sensor. No device migration, dislocation or serious device-related complications occurred. A total of 367 comparisons were included in the IOP agreement analysis. The overall mean difference between GAT and EYEMATE-SC measurements was $1.31 \mathrm{~mm} \mathrm{Hg}$ (lower limit of agreement (LoA) $7.55 \mathrm{~mm} \mathrm{Hg}$; upper LoA -4.92 mm $\mathrm{Hg}$ ). The maximum difference of $2.5 \mathrm{~mm} \mathrm{Hg} \pm 3.96$ (LoA 0.30-2.29) was reached on day 10 and continuously improved to an agreement of $-0.15 \mathrm{~mm}$ $\mathrm{Hg} \pm 2.28$ (LoA -1.24 to 0.89) after 6 months. Accordingly, the percentage of eyes within an IOP difference of $\pm 5 \mathrm{~mm} \mathrm{Hg}$ improved from $78 \%$ (day 3 ) to $100 \%$ (6 months).

Conclusions After 6 months, the EYEMATE-SC sensor was safe and well tolerated, and allowed continual IOP monitoring.

Trial registration number NCT03756662.

\section{INTRODUCTION}

Intraocular pressure (IOP) is the only risk factor for glaucoma that can be modified. Lowering IOP can slow down the progression of glaucomatous optic neuropathy and visual field defects. ${ }^{12}$

Standard tonometry techniques, especially Goldmann applanation tonometry (GAT), require trained personnel and specialised equipment. This limits IOP monitoring to isolated recordings during scheduled visits, usually 3-6 times per year. As a consequence, medical treatment decisions are mostly based on these few random IOP measurements.

However, IOP is highly dynamic and varies throughout the day and from day to day. ${ }^{3}{ }^{4}$ This variability may be an independent risk factor for the development and progression of glaucoma. ${ }^{1}$ IOP fluctuations are influenced by blink, gaze and saccade and telemetric measurements in nonhuman primates showed that IOP fluctuates up to $10 \mathrm{~mm}$ $\mathrm{Hg}$ in the course of the day ${ }^{56}$ Single GAT measurements during normal office hours therefore risk to miss the true peak and trough of the diurnal curve. $^{78}$ For this reason, continual IOP measurement is desirable for glaucoma monitoring.

Sulcus implanted wireless telemetric sensors were shown to be generally safe and to provide long-term reliable IOP measurements. ${ }^{9-11}$ However, several disadvantages can accompany ciliary sulcus implantation, including iris chafing and atrophy, pupillary distortion and pigment dispersion. ${ }^{9} 12$ The restriction to pseudophakic or cataract patients excludes younger patients for whom long-term IOP monitoring is particularly important.

In response to these issues, we have developed a miniaturised telemetric IOP sensor for implantation into suprachoroidal space (EYEMATE-SC, Implandata), which enables a wireless on-demand readout of continuous IOP values via an external handheld reader. In a preceding animal study suprachoroidal implantation proved to be a simple and complication-free procedure, and the device showed good long-term biocompatibility and promising agreement between telemetric and direct intracameral IOP manometry. ${ }^{12}$

The aim of this study was to investigate the safety and performance of the EYEMATE-SC suprachoroidal sensor and the accuracy of its IOP measurements in patients with open-angle glaucoma (OAG) undergoing simultaneous non-penetrating glaucoma surgery (NPGS).

\section{METHODS}

\section{Study design}

First-in-man, prospective, open-label, single-arm, multicentre clinical investigation to assess the safety, tolerability and performance of the EYEMATE-SC suprachoroidal pressure sensor system in patients with OAG undergoing NPGS. 


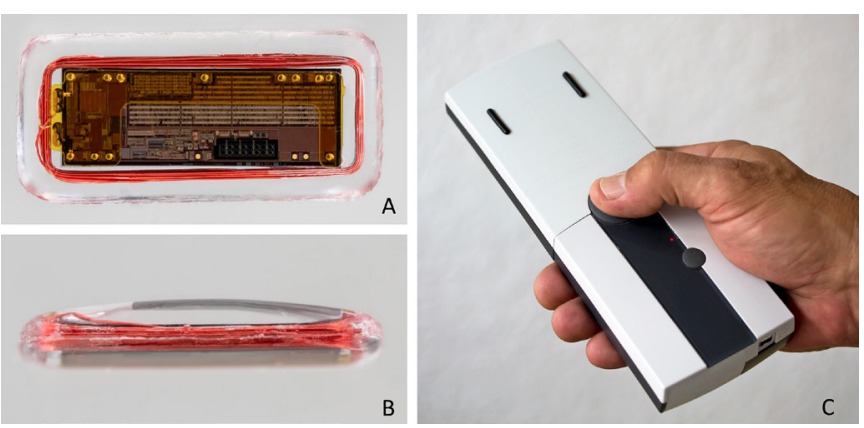

Figure 1 Photograph of the EYEMATE-SC system. (A) Front view (vitreous site) depicting the sensor chip with integrated capacitive pressure sensor membranes and the wire-wound gold microcoil for power and digital data transmission. (B) Angular view with the sclera site up. Note the lenticularly rounded back surface to adapt to the curved scleral shape. (C) Mesograph read-out device.

The study was conducted at five investigational sites in two countries.

The primary objective was to evaluate the safety, tolerability and performance of the EYEMATE-SC system during implantation and throughout a 6-month follow-up period.

The secondary objective of the study was to evaluate the level of agreement between measurements with GAT and the EYEMATE-SC system.

\section{Study patients}

The study included 24 patients of legal age with OAG who were scheduled for NPGS. The medical indication for NPGS had to be given irrespectively of study participation. Potential study patients had given their consent to NPGS before joining the study. Female patients had to have a negative pregnancy test performed prior to surgery. Only one eye per patient was eligible for treatment, although both eyes could be screened for inclusion.

Exclusion criteria comprised neovascular and angle-closure glaucoma, prior glaucoma surgery and other ocular surgery procedures within 6 months (cataract surgery within 3 months) prior to EYEMATE-SC implantation, myopia, axial length $<22 \mathrm{~mm}$ or $>26 \mathrm{~mm}$, or evidence of ocular diseases other than glaucoma. Also, patients with other active medical implants in the head/ neck region or a serious generalised disease were excluded.

\section{Description of device}

The EYEMATE-SC system consists of a cushion-shaped sensor device for permanent implantation into the suprachoroidal space and an external handheld reader device (Mesograph) (see figure 1).

The EYEMATE-SC sensor bears the same microelectromechanical system as the EYEMATE-IO implant, ${ }^{7-9} 11$ although the implant is miniaturised in its final dimensions $(7.5 \times 3.5 \mathrm{~mm}$ and an outwardly decreasing thickness of $1.3 \mathrm{~mm}$ at the centre and $0.9 \mathrm{~mm}$ in the periphery). The surface shape of the implant is lenticularly rounded to smoothly adapt to the curved scleral shape (figure 1A,B).

The custom chip (application-specific integrated circuit, ASIC) integrates pressure and temperature sensors, identification and analog-to-digital encoders, and a telemetry unit. The ASIC is bonded to a wire-wound gold microcoil, and hermetically encapsulated in validated medical-grade silicone rubber material. Owing to the nature of metals contained in the device (gold and aluminium), it is entirely non-magnetic and has been tested for MRI safety.

The Mesograph consists of a power source, a coil for the electromagnetic field supplying the sensor with power, and an antenna for transmitting the signals (figure 1C). IOP is displayed on the LED display of the reader. For a measurement, the system collects and averages 10 samples. The reader can store up to 3000 IOP readings, which can be transferred wired or wirelessly through a Global System for Mobile Communications module.

Each sensor is calibrated to be highly accurate before sterilisation and packaging and is checked for plausible readings immediately before implantation. The implant is tested and certified for electromagnetic and radiation safety according to the requirements of the European medical device law.

\section{Surgical technique}

The EYEMATE-SC implant can be placed either as a stand-alone procedure or in combination with glaucoma surgery. In this study, we included eyes with scheduled NPGS (canaloplasty or deep sclerectomy). The rational is that the access to suprachoroidal space created during the NPGS can easily be used for placing the EYEMATE-SC sensor, so no additional preparation is needed.

After conjunctival peritomy, a $5 \times 5 \mathrm{~mm}$ superficial scleral flap is created. Below this, a deep scleral flap $(4 \times 4 \mathrm{~mm})$ is prepared. Depending on the surgeon's preference, the second flap is either prepared completely until the choroid is exposed (100\% thickness technique, figure $2 \mathrm{~A}-\mathrm{C}$ ) or a thin scleral lamella of $10-50 \mu \mathrm{m}$ is preserved ( $90 \%$ thickness technique, figure $2 \mathrm{D}-\mathrm{F})$. The dissection is continued across the scleral spur to open Schlemm's canal (SchC). After SchC is deroofed a trabecular-Descemet window is created. The deep scleral flap is excised and juxtacanalicular TM is peeled. SchC may additionally be dilated with viscoelastics (OVD; viscocanalostomy) or probed with a microcatheter and tensioned with a $10-0$ prolene suture (canaloplasty).

To implant the sensor, suprachoroidal space is accessed either through the window (100\% thickness technique) or through a $5 \mathrm{~mm}$ incision in the remaining scleral lamella (90\% thickness technique). The choroid is separated from the sclera by means of OVD, and the EYEMATE-SC implant is carefully inserted into the suprachoroidal space using padded implantation forceps and avoiding contact with the sensor's ASIC. Afterward, the superficial scleral flap and the conjunctiva are closed and sutured.

\section{Follow-up examinations}

Follow-up examinations were performed on days 1, 3, 10, 30, 90 and 180. An ophthalmological examination including bestcorrected visual acuity, slit-lamp biomicroscopy, funduscopy and tonometry was performed at each visit, and all findings were recorded. External eye photography and optical coherence tomography (OCT) of the anterior segment were performed at baseline visit and days 10,30,90 and 180. Tonometry was performed using GAT and EYEMATE-SC at each visit. EYEMATE measurements were performed by the patients. GAT was performed with two measurements; in case of a difference of more than $2 \mathrm{~mm} \mathrm{Hg}$, a third GAT measurement was required. In order to avoid operator/investigator bias, GAT measurements were taken directly prior to EYEMATE measurements to ensure the investigator is unaware of the EYEMATE result.

All patients received the reading unit for long-term selftonometry and were encouraged to measure IOP at home regularly. 

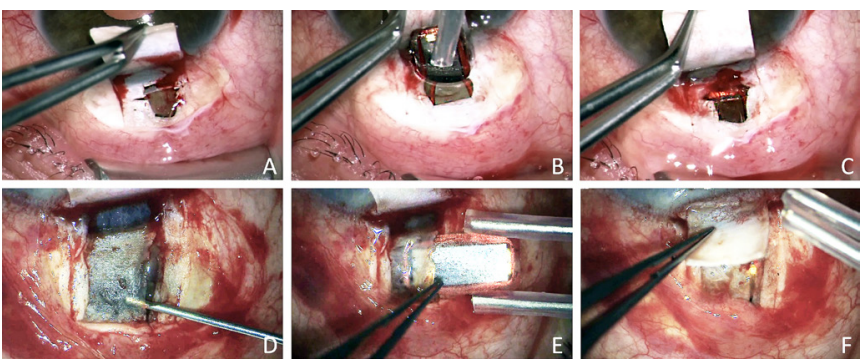

Figure 2 Two techniques for suprachoroidal implantation of the EYEMATE-SC sensor device. (A-C) Preparation with 100\% thickness technique $^{13}$ : the deep flap is prepared until the choroid is completely exposed (A). At the end of NPGS, viscoelastics are injected into the suprachoroidal space, and the EYEMATE-SC sensor is carefully inserted using padded implantation forceps (B). The sensor is buried in the suprachoroidal space, acting as a spacer for suprachoroidal drainage. (D-F) Preparation with $90 \%$ thickness technique: the deep flap is prepared und a thin scleral lamella preserved; the choroid is not exposed (D). At the end of NPGS, a $5 \mathrm{~mm}$ incision in the remaining thin scleral lamella is made (D) and the EYEMATE-SC sensor is inserted into the suprachoroidal space $(E)$. At the end of the procedure, the sensor is buried in the suprachoroidal space without additional suprachoroidal drainage (F). NPGS, non-penetrating glaucoma surgery.

\section{Statistical analysis}

The safety population included all subjects in whom EYEMATE-SC implantation was attempted. The per-protocol set comprised all subjects in whom the sensor was successfully implanted and for whom the full dataset was available until month 6.

Bland-Altman plots were used to evaluate the mean difference and 95\% limit of agreement (LoA) in IOP measurements between the averages of GAT and EYEMATE-SC at each study visit. ${ }^{13}$ The Pearson correlation coefficient $r$ was used to measure the strength of the agreement between GAT and EYEMATE-SC on the scatter plot. For performance analysis, the mean difference in the measurements between the two methods on the Bland-Altman plot was the estimate of the fixed bias. The probability distribution of the difference of the paired measurements grouped within $1 \mathrm{~mm} \mathrm{Hg}$ was compared with the primary objective of the accepted $70 \%$ of the measurements to agree between $\pm 5 \mathrm{~mm} \mathrm{Hg}$. This limit was derived from ANSI Z80.10. Statistical analysis was performed using the statistical software Stata V.14.2 (StataCorp). A p $\leq 0.05$ was considered statistically significant.

\section{RESULTS}

\section{Patient characteristics}

The 24 patients who met the inclusion criteria originated from 5 centres and 7 surgeons performed the surgeries. The mean age was $65.1 \pm 10.2$ years, $50 \%$ were women. Fifty per cent were right eyes, 8 eyes were pseudophakic and 14 phakic, 2 eyes underwent combined cataract surgery. Primary OAG was the predominant diagnosis $(n=20)$, followed by pseudoexfoliation $(n=2)$, pigment dispersion $(n=1)$ and uveitic glaucoma $(\mathrm{n}=1)$. Mean preoperative IOP was $19.4 \pm 5.4 \mathrm{~mm} \mathrm{Hg}$ (range: $12-37 \mathrm{~mm} \mathrm{Hg}$ ) with a mean number of antiglaucomatous medication of $2.8 \pm 0.8$ (range: $0-4$ ).

The patients underwent the procedure with either canaloplasty $(n=15)$ or deep sclerectomy $(n=9)$. EYEMATE-SC implantation was successful in all cases, and all implanted sensors remained in the eye during follow-up. All patients completed the 3-month
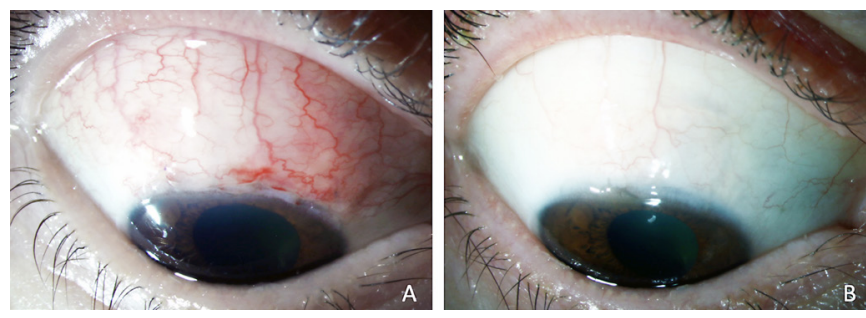

Figure 3 Postoperative anterior segment photography of a patient at day 10 (A) and at day 90 (B).

follow-up, and 21 patients completed the 6-month follow-up, 3 patients missed the follow-up due to COVID-19.

\section{Safety}

The implantation of the EYEMATE-SC succeeded without complications in all eyes. No intraoperative injury of the choroid or bleeding from the entry site were reported. In all cases the sensor could be implanted with the right orientation (ASIC towards the choroid).

During the first 6 months, no serious or sight-threatening complications occurred. Figure 3 shows the postoperative anterior segment photography of a patient at day 10 and 90, respectively.

The most frequent postoperative complication was hyphaema, which occurred in nine eyes (seven after canaloplasty, two after deep sclerectomy) and resolved spontaneously in all cases. Two patients had superficial punctate keratitis for 3 weeks. One patient showed early postoperative leakage, one patient had choroidal detachment and hypotony for 3 weeks, which resolved without sequelae. One patient complained about postoperative photopsia for 5 weeks. Three patients reported touch sensitivity, two reported slight pain in the operative area, and one had intermittent headaches. The touch sensitivity was assessed as possibly device related. All other AEs were assessed and determined to be caused by the surgical intervention itself. No serious AEs related to the EYEMATE-SC sensor were reported.

The position, orientation and distance of the sensor from the scleral spur, which were measured by anterior segment OCT and $50 \mathrm{MHz}$ ultrasound biomicroscopy, showed complete spatial stability. There were no reports of device migration, rotation or dislocation (figure 4). In one patient, the implant, partially inserted into the suprachoroidal space at the end of surgery with approximately $1 / 6$ th remaining in the deep scleral lake, slipped back early postoperatively into the suprachoroidal space and remained stable there, as intended.

\section{Performance}

Measurement agreement

No permanent device malfunction occurred. Bland-Altman plots were used for analysing the agreement between the measurement with GAT and EYEMATE-SC throughout the follow-up visits (figure 5). A total of 405 comparisons were included in the analysis. The overall mean measurement difference for all pressure sensors and all follow-up measurements was $1.31 \mathrm{~mm}$ $\mathrm{Hg}$ (lower LoA $7.55 \mathrm{~mm} \mathrm{Hg}$; upper LoA -4.92 mm Hg).

An analysis over time shows that during the first month the EYEMATE-SC IOP values were significantly higher than the GAT measurements, with a maximum difference of $2.5 \mathrm{~mm}$ $\mathrm{Hg} \pm 3.96$ (LoA 0.30-2.29) at day 10. In the further course of the study, the overall bias between the two methods significantly declined, reaching an satisfying agreement after 3 and 6 months. 

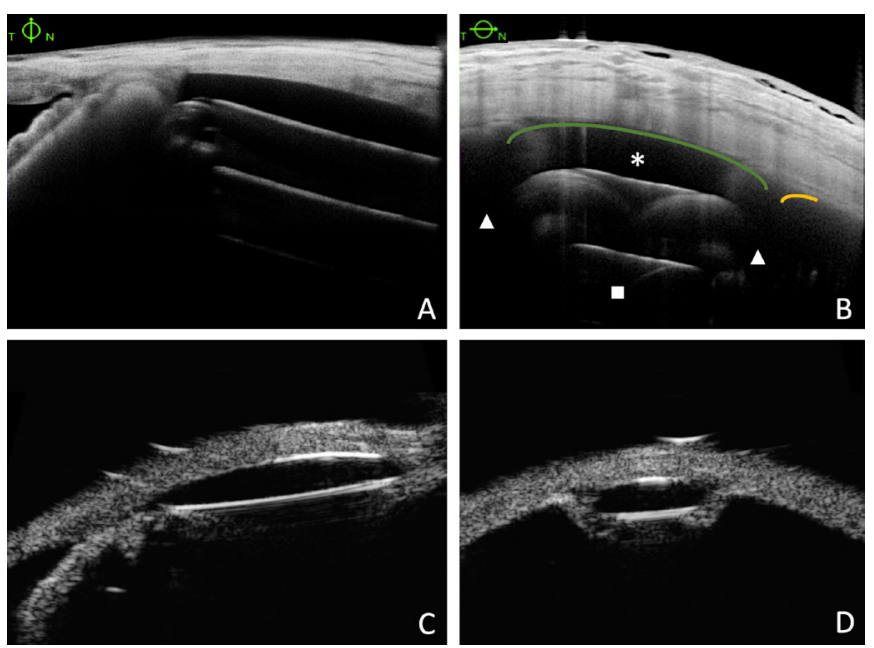

Figure 4 Multimodal imaging 6 months after suprachoroidal implantation of the EYEMATE-SC sensor using AS-OCT $(A, B)$ and 50 $\mathrm{MHz}$ ultrasound biomicroscopy (UBM, $(C, D)$ of the same patient with meridional $(A, C)$ and limbus-parallel $(B, D)$ imaging. It shows the sensor remaining stable underneath the choroid (orange line) at the site of implantation. Of note, AS-OCT visualises the microelectronics carrier substrate $(\boldsymbol{\square})$ and the gold coil $(\boldsymbol{\Lambda})$, but not the silicone encapsulation ( ${ }^{*}$ and green line). in contract, UBM depicts the lenticularly rounded silicone encapsulation smoothly adapting to the curved scleral shape. AS-OCT, anterior segment optical coherence tomography.

Correspondingly, the percentage of eyes where the difference in the IOP measurement between GAT and EYEMATE-SC was within $\pm 5 \mathrm{~mm} \mathrm{Hg}$ improved from $78 \%$ (day 3) to $100 \%$ (6 months).

Figure 6 shows a scatter plot of all paired GAT/EYEMATE-SC comparisons. It reveals that the deviation is not prominent in a certain IOP level but is evenly and closely distributed around the idealised zero error line (dotted line).

Postoperatively, a transient increase of astigmatism was reported. The mean refractive cylinder, irrespective of the axis, increased from $1.1 \mathrm{dpt}$ (dioptres) at the screening visit to $2.1 \mathrm{dpt}$ (day 3), and $2.3 \mathrm{dpt}$ (day 10) and returned to $1.4 \mathrm{dpt}$ (day 30) and $1.3 \mathrm{dpt}$ (day 180) (see table 1). This transiently increased astigmatism influences the accuracy of GAT and the Bland-Altman LoA shows a better agreement between GAT and the EYEMATE-SC measurement if the first month is excluded (figure 7). At the 6-month study visit, all eyes achieved agreement within the targeted $\pm 5 \mathrm{~mm} \mathrm{Hg}$ and a mean difference of $-0.15 \mathrm{~mm} \mathrm{Hg} \pm 2.28 \mathrm{SD}$ (LoA -1.24 to 0.89 ).

\section{DISCUSSION}

The 6-month interim evaluation shows that the EYEMATE-SC sensor is a safe and well-tolerated suprachoroidal implant that allows continuous IOP monitoring of glaucoma patients.

Once implanted, patients can measure their IOP without contact by simply bringing the external reader next to the eye to enable electromagnetic coupling of the sensor. Patients can automatically create their individual IOP profile to disclose short-term and long-term fluctuations enabling the ophthalmologist to adapt the therapy. The measured values correspond to the IOP independent of the corneal biomechanics, ${ }^{14}$ and the active involvement of the patients as well as the direct treatment response are suitable to improve the therapy adherence in glaucoma patients. ${ }^{15}$

The preceding generation of telemetric sensors implanted in the sulcus (EYEMATE-IO) were generally safe and reliable. ${ }^{9-11}$ However, their use is restricted to pseudophakic or cataract patients and excludes patients with a narrow chamber angle. Furthermore, the bulky sensor ring needs a large corneoscleral incision and causes excessive manipulation during sulcus implantation. ${ }^{11}$

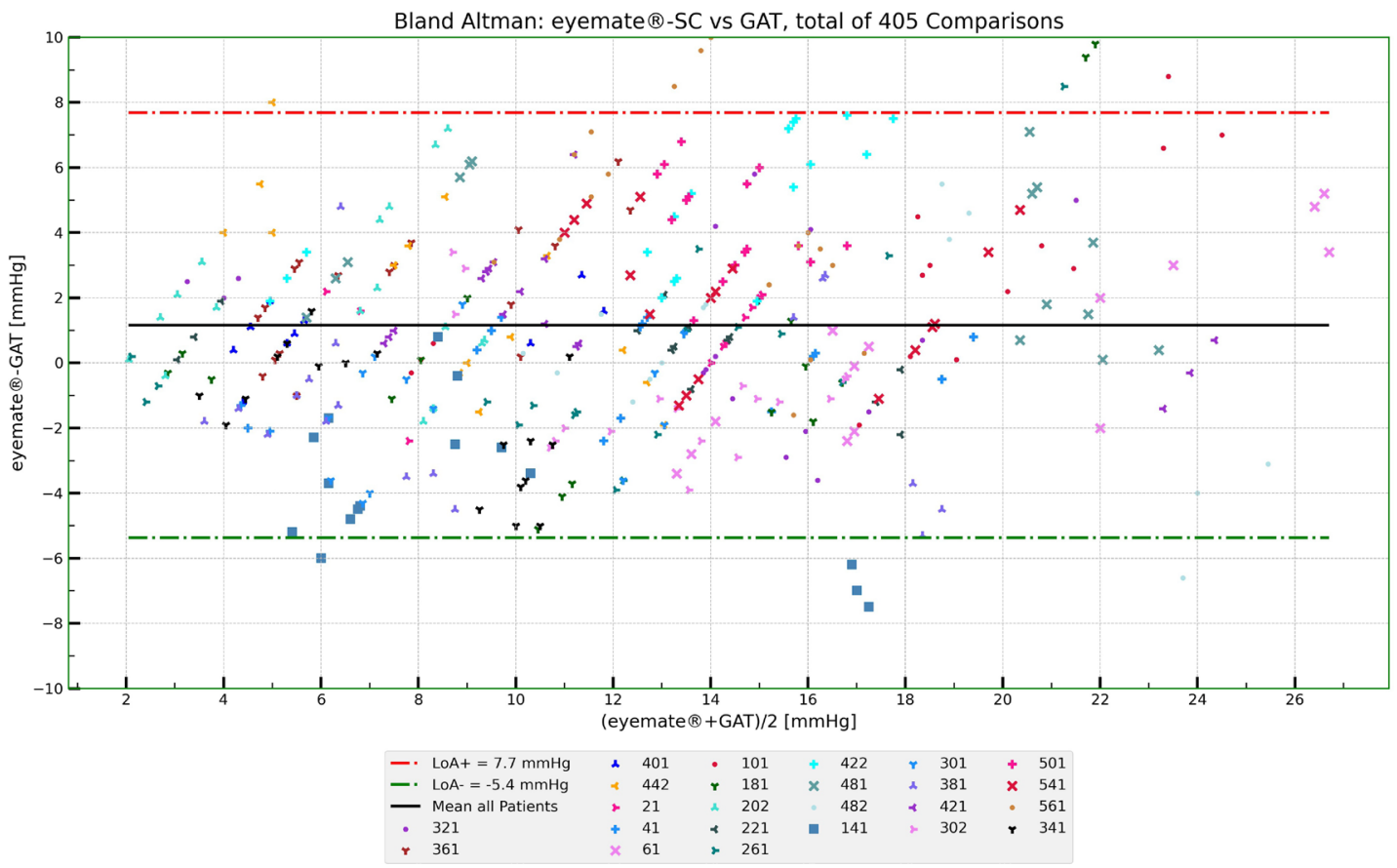

Figure 5 Bland-Altman analysis of agreement between GAT and EYEMATE-SC (95\% Cl). All data points within 180 days (405), available postoperative study from day 1 to day 180 are plotted. The bold black line represents the average over all comparisons; red and green dashed-dotted lines represent the upper and lower level of agreement (LoAs, mean difference $\pm 1.96 \mathrm{SDs}, 95 \% \mathrm{CI}$ ). GAT, Goldmann applanation tonometry. 


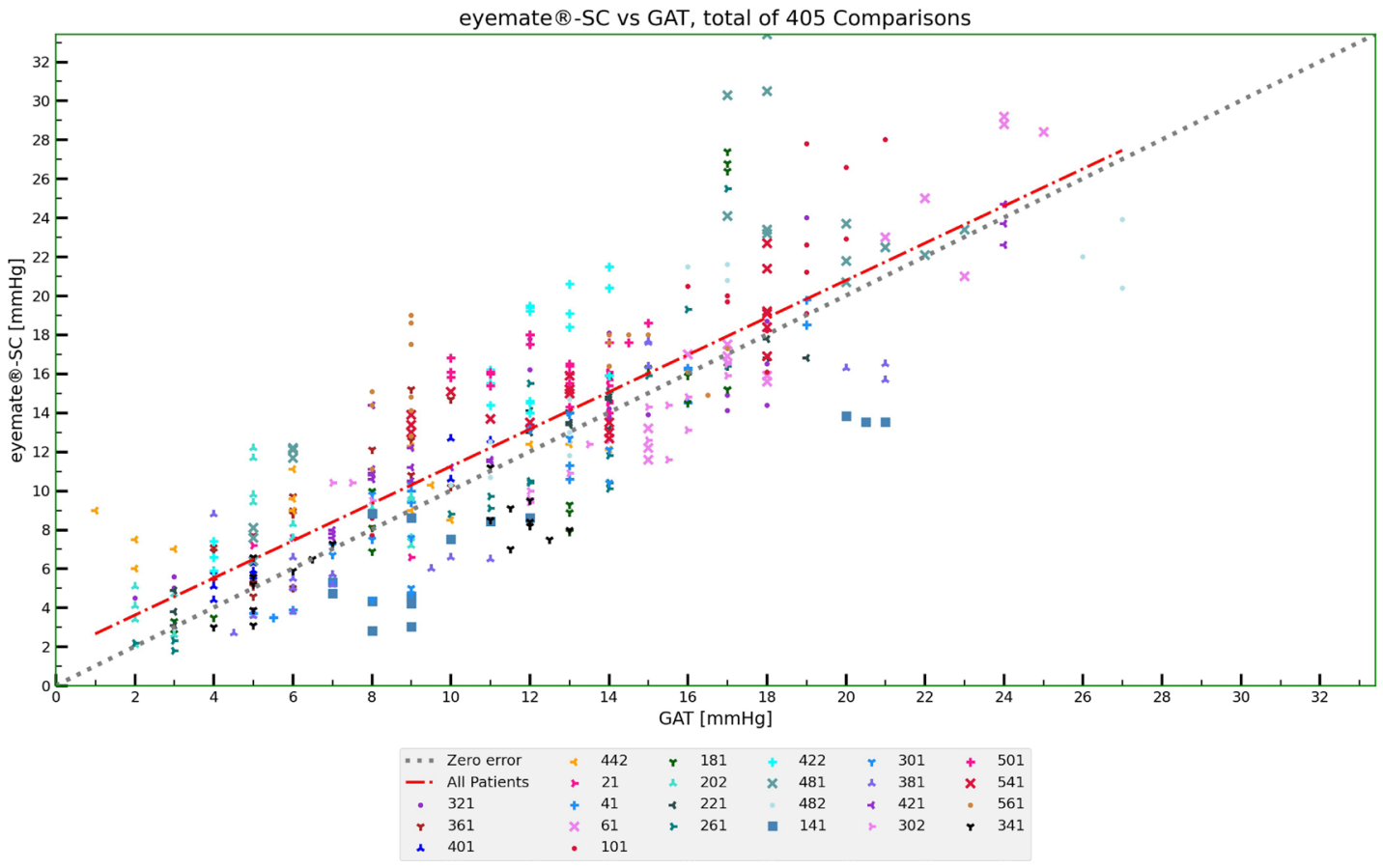

Figure 6 Scatter plot of all paired GAT/EYEMATE-SC comparisons. It reveals that the deviation is evenly and closely distributed around the idealised zero error line (dotted line). The best fit linear regression line (red dash dot) shows a slight positive bias towards lower IOP values. GAT, Goldmann applanation tonometry; IOP, intraocular pressure.

This suprachoroidal approach was developed to solve these limitations. The EYEMATE-SC system consists of a miniaturised IOP sensor that is implanted into suprachoroidal space. It can be used independent of the lens status and is also suitable for younger glaucoma patients for whom continuous IOP monitoring is particularly important. Implantation can also be combined with trabeculectomy. The long-term goal is a further miniaturised device for very small incision implantation in a stand-alone procedure preferably in one of the lower quadrants to leave space in the upper ones for glaucoma surgery.

The reported nine cases of hyphaema were judged to be surgery related but not device related. Hyphaema is a known side effect of NPGS, especially for canaloplasty. The intraoperative occurrence is caused by blood reflux from SchC and the collector channels. Usually hyphaema is resorbed spontaneously. Some authors even presume that it is a sign of surgical success regarding IOP development. ${ }^{16}{ }^{17}$ Also after deep sclerectomy hyphaema is reported to occur in up to $21 \%{ }^{18}$ It could result from perforation of Descemet's membrane and subsequent

Table 1 Deviation between EYEMATE-SC and Goldmann tonometry presented with corneal astigmatism values at the different time points

\begin{tabular}{|c|c|c|c|c|c|c|c|}
\hline Visit & $\begin{array}{l}\text { Mean of } \\
\text { paired } \\
\text { delta } \\
(\mathrm{mm} \mathrm{Hg})\end{array}$ & $\begin{array}{l}\text { Lower LoA } \\
(\mathrm{mm} \mathrm{Hg})\end{array}$ & $\begin{array}{l}\text { Upper LoA } \\
(\mathrm{mm} \mathrm{Hg})\end{array}$ & $\begin{array}{l}\text { Subj. } \\
\text { CYL } \\
\text { mean } \\
\text { (dpt) }\end{array}$ & $\begin{array}{l}\text { Subj. } \\
\text { CYL } \\
\text { STD } \\
\text { (dpt) }\end{array}$ & $\begin{array}{l}\text { Subj. } \\
\text { CYL } \\
\min \\
\text { (dpt) }\end{array}$ & $\begin{array}{l}\text { Subj. } \\
\text { CYL } \\
\max \\
\text { (dpt) }\end{array}$ \\
\hline Screening & - & - & - & -1.1 & 1.5 & -4.8 & 0.0 \\
\hline D1 & 1.5 & -3.3 & 6.3 & -1.3 & 1.3 & -5.0 & -0.3 \\
\hline D3 & 1.2 & -6.2 & 8.7 & -2.1 & 1.9 & -7.8 & -0.3 \\
\hline D10 & 2.5 & -5.5 & 10.5 & -2.3 & 1.6 & -6.5 & -0.3 \\
\hline D30 & 1.9 & -4.7 & 8.5 & -1.4 & 1.2 & -5.3 & -0.3 \\
\hline D90 & 0.1 & -5.5 & 5.6 & -1.4 & 1.2 & -5.5 & 0.0 \\
\hline
\end{tabular}

CYL, cylinder; LoA, limit of agreement; STD, standard deviation. bleeding under the conjunctiva or blood flow from iris vessels or ciliary processes. ${ }^{19}$

Of note, no choroid-associated problems such as choroidal injury or bleeding, macular oedema or suprachoroidal migration of the implant were reported during the study course. This confirms the results of previous experiments on rabbits, which showed that the suprachoroidal space can be easily expanded with OVD to implant the sensor comfortably without the risk of choroidal injury. ${ }^{12}$ Besides, other surgical methods also place glaucoma or retinal implants in suprachoroidal space. ${ }^{20-24}$

During the study several centres in Germany and Switzerland were involved, and none of the surgeons found the implantation difficult or reported problems. This contrasts with the EYEMATE-IO, where a significant learning curve for the sulcusbased implantation was reported which might partially explain the number of implantation-related AEs. ${ }^{11}$

If YAG goniopuncture is needed after NPGS to enhance the pressure-lowering effect, a loss of sensor function is physically unlikely. The experiences we have made with YAG capsulotomy in the Eyemate IO study as well as two cases where a YAG goniopuncture was performed after Eyemate-SC implantation show that after recalibration the sensor function was flawless.

One of the main advantages of IOP sensors is that they are independent of corneal biomechanics. While corneal-surfacebased tonometry techniques only measure relative dimensional changes of the eye with questionable validity, ${ }^{25} 26$ intraocular sensors directly measure the absolute IOP. In rabbit experiments, the suprachoroidal sensor showed a high agreement between the telemetrically assessed IOP values and the effective intracameral pressure assessed by direct cannulation of the anterior chamber with a manometry probe. ${ }^{12}$ This confirms that intraocular sensors are largely independent of external factors such as corneal thickness, rigidity and astigmatism, which in particular compromise the validity of GAT. ${ }^{27}$ 


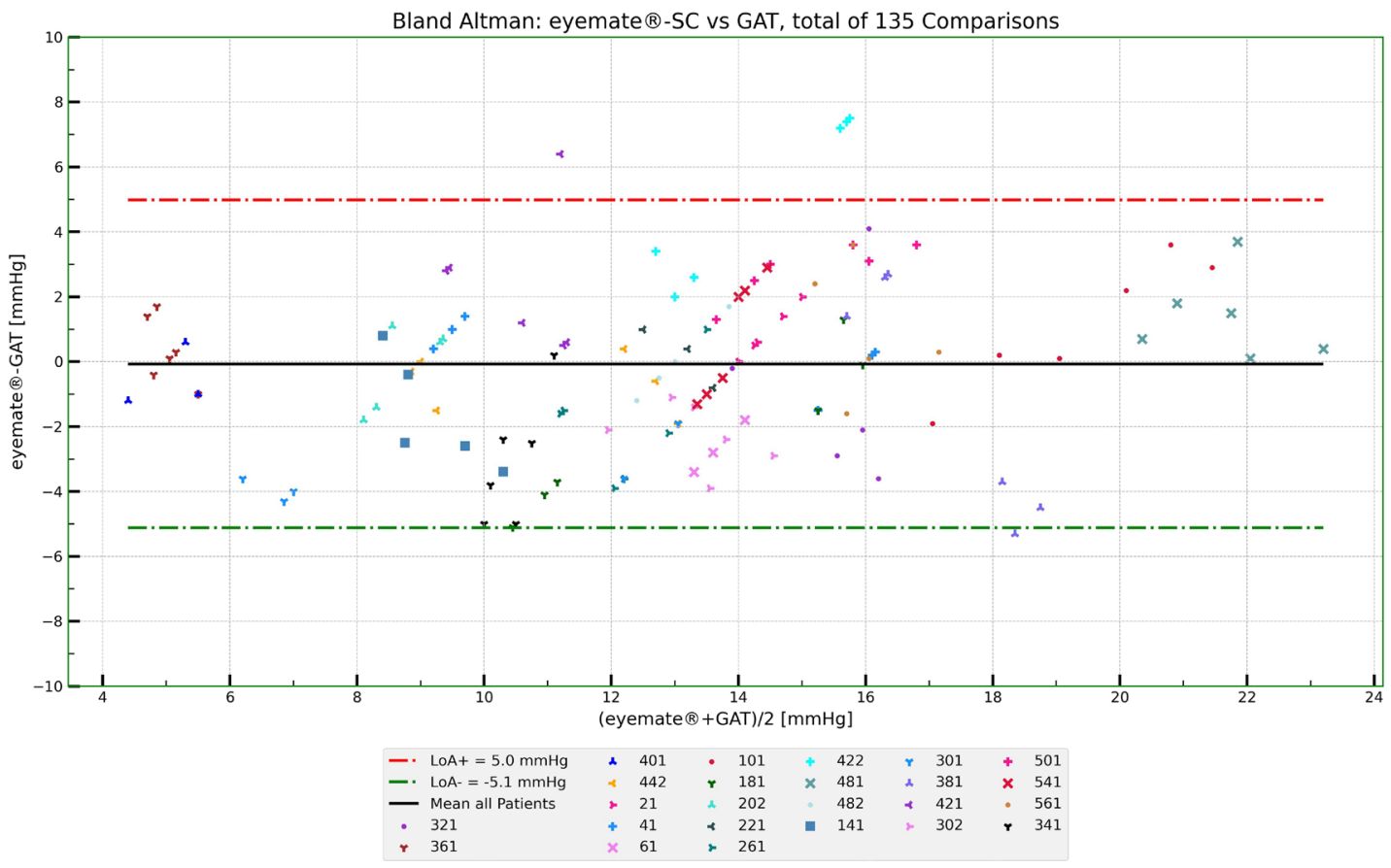

Figure 7 Bland-Altman analysis of agreement between Goldmann applanation tonometry (GAT) and the EYEMATE-SC (95\% CI). Only data available from day 90 and day 180 are plotted to exclude the postoperative interval of increased astigmatism. After 3 months, the lower limit of agreement (LOA) significantly improved to LOA $-4.6 /+4.9 \mathrm{~mm} \mathrm{Hg}$. The bold black line represents the average over all comparisons; red and green dashed-dotted lines represent the upper and lower LOA (mean difference + /-1.96 SDs).

In our study, transient deviations were observed in the agreement between GAT and the telemetric EYEMATE-SC IOP within the first few weeks after implantation; the deviations normalised after 3 months. This was concomitant but not directly correlated with a temporarily observed significant increase in postoperative astigmatism due to the scleral flap sutures of the NPGS (see table 1).

It is known that GAT tends to underestimate the IOP in eyes with irregular ${ }^{2829}$ or significantly increased central corneal thickness, and therefore, its reliability should be interpreted carefully in those patients. ${ }^{30}$

As there is no direct correlation between the increase in astigmatism and the difference between EYEMATE-SC and Goldmann values, there are probably other factors than astigmatism that influence the accuracy of Goldmann measurement in the early postoperative phase. For instance, it is known that the accuracy of GAT decreases in higher IOP levels measuring false-low values. ${ }^{31-33}$ This is in accordance with the ARGOS-02 (EYEMATE-IO) trial showing an increasing bias between GAT and telemetric IOP values at higher pressure levels. Furthermore, our experience from clinical practice shows that it is very difficult to achieve reliable IOP values with GAT in hypotonic eyes. And in the first 2 weeks after a canaloplasty or deep sclerectomy, hypotony is common as the sutures are not immediately watertight.

One might, therefore, assume that telemetric IOP measurements achieve a higher validity than GAT, especially in eyes with altered corneal biomechanics and at abnormal IOP levels. However, without comparing the values with real-time IOP measurement using intraocular probes this assumption remains merely speculative.

Limitations of this study include the relatively small and heterogeneous patient population. The follow-up period is still relatively short, but the 6-month interim analysis was intended to focus on safety and performance in the postoperative healing process. Also, potential explantability of the implant has only been proven in human autopsy eyes and in rabbit experiments. Further observations are necessary to assess the long-term safety, performance and reliability of the implant and the system.

In summary, the EYEMATE-SC is the first suprachoroidal sensor for telemetric IOP measurement. It has been successfully and safely implanted in all 24 patients during simultaneous NPGS. The 6-month evaluation showed no severe complications, malfunctions or implant migration. The suprachoroidal sensor allows continuous telemetric IOP self-measurement, even under conditions where the validity of the GAT may be limited.

Acknowledgements The authors thank the EYEMATE-SC study investigators and clinical research teams for their dedication and support.

Collaborators The EYEMATE-SC study group: Further Study Team: Eye Clinic Sulzbach: Arno Haus, MD, Eugen Reifschneider, MD, Annekatrin Rickmann, MD, Philip Wakili, MD, Caroline Emmerich, MD, Caroline Hoogmartens, MD, Kristina Lambert, MD, Kai Januschowski, MD University Eye Clinic Bochum: Matthias Elling,MD, Tim Schultz, MD, Inga Kersten-Gomez, MD University Medical Center Mainz: Katrin Lorenz, MD, Bert Glers, MD, Anna Beck, MD Ludwig-Maximilians-University Munich: Matthias Nobl, MD, Sigrid Freissinger, MD.

Contributors The authors are justifiably credited with authorship, according to the authorship criteria. In detail: PS, HLR and A-MS - data analysis and interpretation, manuscript preparation and revision. PS, KM, BD, AM, EMH, MM and RNWresearch design and execution, data acquisition, revision and final approval.

Funding This study received support from Implandata Ophthalmic Products, Hannover, Germany, which produced and provided the medical devices under investigation.

Competing interests PS has a pending international patent for the EYEMATESC system (PCT/EP2015/062976). EMH reports grants from Allergan, Novartis, Heidelberg Engineering, Santen, Thea outside the submitted work; KM reports grants from Implandata (C) and non-related financial disclosures from Santen (C), Fabrinal (C), Alcon (S), Allergan (S), Optovue (S); New World Medical (C), Oertli (C) MM reports grants from Implandata (C) and non-related financial disclosures from Acufocus, Allergan, Bausch and Lomb, Bayer, DORC, Heidelberg Engineering, Iridex, Novartis, Oculus, Optos, Roche, Santen, Topcon, Zeiss. HLR reports consulting fees from Santen and Allergan and research support from Carl Zeiss Meditec 
Patient consent for publication Not applicable.

Ethics approval The study protocol was approved by the ethics committee of Saarland (ID:CIV-18-07-025065) and followed the tenets of the Declaration of Helsinki.

Provenance and peer review Not commissioned; externally peer reviewed.

Data availability statement Data may be obtained from a third party and are not publicly available. Not applicable.

Open access This is an open access article distributed in accordance with the Creative Commons Attribution Non Commercial (CC BY-NC 4.0) license, which permits others to distribute, remix, adapt, build upon this work non-commercially, and license their derivative works on different terms, provided the original work is properly cited, appropriate credit is given, any changes made indicated, and the use is non-commercial. See: http://creativecommons.org/licenses/by-nc/4.0/.

ORCID iDs

Harsha Laxmana Rao http://orcid.org/0000-0001-5866-9856

Anna-Maria Seuthe http://orcid.org/0000-0002-3195-6537

\section{REFERENCES}

1 Weinreb RN, Aung T, Medeiros FA. The pathophysiology and treatment of glaucoma: a review. JAMA 2014:311:1901-11.

2 Schuster AK, Erb C, Hoffmann EM, et al. The diagnosis and treatment of glaucoma. Dtsch Arztebl Int 2020:117:225-34.

3 Realini T, Weinreb RN, Wisniewski SR. Diurnal intraocular pressure patterns are not repeatable in the short term in healthy individuals. Ophthalmology 2010;117:1700-4.

4 Realini T, Weinreb RN, Weinreb N, et al. Short-term repeatability of diurnal intraocular pressure patterns in glaucomatous individuals. Ophthalmology 2011;118:47-51.

5 Coleman DJ, Trokel S. Direct-recorded intraocular pressure variations in a human subject. Arch Ophthalmol 1969;82:637-40.

6 Downs JC, Burgoyne CF, Seigfreid WP, et al. 24-hour IOP telemetry in the nonhuman primate: implant system performance and initial characterization of IOP at multiple timescales. Invest Ophthalmol Vis Sci 2011:52:7365-75.

7 Liang SY-W, Lee GA, Shields D. Self-tonometry in glaucoma management--past, present and future. Surv Ophthalmol 2009;54:450-62.

8 Mansouri K, Shaarawy T. Continuous intraocular pressure monitoring with a wireless ocular telemetry sensor: initial clinical experience in patients with open angle glaucoma. Br J Ophthalmol 2011;95:627-9.

9 Koutsonas $\mathrm{A}$, Walter $\mathrm{P}$, Roessler $\mathrm{G}$, et al. Implantation of a novel telemetric intraocular pressure sensor in patients with glaucoma (argos study): 1-year results. Invest Ophthalmol Vis Sci 2015;56:1063-9.

10 Koutsonas A, Walter P, Roessler G, et al. Long-term follow-up after implantation of a telemetric intraocular pressure sensor in patients with glaucoma: a safety report. Clin Exp Ophthalmol 2018:46:473-9.

11 Choritz L, Mansouri K, van den Bosch J, et al. Telemetric measurement of intraocular pressure via an implantable pressure Sensor-12-Month results from the ARGOS-02 trial. Am J Ophthalmol 2020;209:187-96.

12 Mariacher S, Ebner M, Januschowski K, et al. Investigation of a novel implantable suprachoroidal pressure transducer for telemetric intraocular pressure monitoring. Exp Eye Res 2016;151:54-60
13 Bland JM, Altman DG. Measuring agreement in method comparison studies. Stat Methods Med Res 1999:8:135-60.

14 Todani A, Behlau I, Fava MA, et al. Intraocular pressure measurement by radio wave telemetry. Invest Ophthalmol Vis Sci 2011;52:9573-80.

15 Sleath B, Blalock S, Covert D, et al. The relationship between glaucoma medication adherence, eye drop technique, and visual field defect severity. Ophthalmology 2011;118:2398-402.

16 Grieshaber MC, Pienaar A, Olivier J, et al. Canaloplasty for primary open-angle glaucoma: long-term outcome. Br J Ophthalmol 2010;94:1478-82.

17 Koch JM, Heiligenhaus A, Heinz C. [Canaloplasty and transient anterior chamber haemorrhage: a prognostic factor?]. Klin Monbl Augenheilkd 2011;228:465-7.

18 Cillino S, Di Pace F, Casuccio A, et al. Deep sclerectomy versus trabeculectomy with low-dosage mitomycin C: four-year follow-up. Ophthalmologica 2008;222:81-7.

19 Mendrinos E, Mermoud A, Shaarawy T. Nonpenetrating glaucoma surgery. Surv Ophthalmol 2008:53:6:592-630.

20 Szurman P, Januschowski K, Boden KT, et al. Suprachoroidal drainage with collagen sheet implant- a novel technique for non-penetrating glaucoma surgery. Graefes Arch Clin Exp Ophthalmol 2018;256:381-5.

21 Seuthe A-M, Ivanescu C, Leers S, et al. Modified canaloplasty with suprachoroidal drainage versus conventional canaloplasty-1-year results. Graefes Arch Clin Exp Ophthalmol 2016;254:1591-7.

22 Ayton LN, Blamey PJ, Guymer RH, et al. First-in-human trial of a novel suprachoroidal retinal prosthesis. PLoS One 2014;9:e115239.

23 Cereda MG, Parolini B, Bellesini E, et al. Surgery for CNV and autologous choroidal RPE patch transplantation: exposing the submacular space. Graefes Arch Clin Exp Ophthalmol 2010:248:37-47.

$24 \mathrm{Ma}$ Z, Han L, Wang C, et al. Autologous transplantation of retinal pigment epitheliumBruch's membrane complex for hemorrhagic age-related macular degeneration. Invest Ophthalmol Vis Sci 2009:50:2975-81.

25 Kakaday T, Hewitt AW, Voelcker NH, et al. Advances in telemetric continuous intraocular pressure assessment. Br J Ophthalmol 2009;93:992-6.

26 Sit AJ. Continuous monitoring of intraocular pressure: rationale and progress toward a clinical device. J Glaucoma 2009;18:272-9.

27 Liu J, Roberts CJ. Influence of corneal biomechanical properties on intraocular pressure measurement: quantitative analysis. J Cataract Refract Surg 2005;31:146-55.

28 Meyenberg A, lliev ME, Eschmann R, et al. Dynamic contour tonometry in keratoconus and postkeratoplasty eyes. Cornea 2008;27:305-10.

29 Rosa N, Cennamo G, Breve MA, et al. Goldmann applanation tonometry after myopic photorefractive keratectomy. Acta Ophthalmol Scand 1998;76:550-4.

30 Ceruti P, Morbio R, Marraffa M, et al. Comparison of Goldmann applanation tonometry and dynamic contour tonometry in healthy and glaucomatous eyes. Eye 2009;23:262-9.

31 McCafferty S, Levine J, Schwiegerling J, et al. Goldmann applanation tonometry error relative to true intracameral intraocular pressure in vitro and in vivo. BMC Ophthalmol 2017;17:215.

32 Whitacre MM, Stein R. Sources of error with use of Goldmann-type tonometers. Surv Ophthalmol 1993;38:1-30.

33 Kniestedt C, Nee M, Stamper RL. Dynamic contour tonometry: a comparative study on human cadaver eyes. Arch Ophthalmol 2004;122:1287-93. 